\title{
Aislamiento e identificación de oomicetos en focos de podredumbre radical de Andalucía y Extremadura
}

\author{
Ruiz Gómez, F.J." ${ }^{*}$, Navarro Cerrillo, R.M. ${ }^{1}$, Lara Gómez, M.A. ${ }^{2}$ y Sánchez-Cuesta, R. ${ }^{1}$ \\ ${ }^{1}$ Grupo Evaluación y Restauración de Sistemas Agrícolas y Forestales (RNM360). \\ Departamento de Ingeniería Forestal, Universidad de Córdoba. Edificio Leonardo Da Vinci, $1^{a}$ Planta. \\ Campus de Rabanales, CT. N-IVa, km 396. CP 14071 Córdoba.
}

${ }^{2}$ IDAF-Centro de Investigaciones Aplicadas al Desarrollo Agroforestal. Edificio Leonardo Da Vinci, Planta baja. Campus de Rabanales, CT. N-IVa, km 396. CP 14071 Córdoba.

*Autor para correspondencia: g72rugof@uco.es

\section{Resumen}

El decaimiento de los Quercus sigue siendo uno de los principales problemas de sanidad forestal en España y Portugal. Es difícil cuantificar los ingentes recursos económicos y humanos destinados a la búsqueda de soluciones, sin que se haya podido atajar el avance de la mortalidad en las dehesas. Aunque se señala a Phytophthora cinnamomi como agente causal de los episodios más graves, otros oomicetos han sido identificados como agentes relacionados con estos procesos de mortalidad, o como patógenos oportunistas o acompañantes. La complejidad de los sistemas forestales influye en la diversidad de patógenos de podredumbre radical en el suelo, por lo que se debe tener en cuenta el efecto agregativo de la interacción de dichos organismos en la podredumbre radical. En el presente trabajo se colectaron muestras de suelo en dehesas de Córdoba, Sevilla y Cáceres, en áreas de afección por decaimiento. El daño del arbolado se evaluó mediante una escala asimilada de Ferreti. Las muestras se cultivaron en medio selectivo, y se identificaron morfológicamente 26 especies diferentes de oomicetos. Phytohthora cinnamomi fue el patógeno que mayor número de aislamientos presentó. El número de especies aisladas en función del grado de daños mostró diferencias significativas, siendo los árboles con grado 2 aquellos en los cuales se identificó el mayor número de especies. La presencia de P. cinnamomi no estuvo asociada significativamente con la diversidad de otras especies.

Palabras clave: Decaimiento, interacción Phytophthora, Pythium, Quercus ilex. 


\section{Introducción}

El decaimiento de los Quercus en el suroeste de la Península Ibérica sigue siendo uno de los principales problemas de sanidad forestal en España y Portugal, problema que viene afectando gravemente a masas de encina (Quercus ilex L.) y alcornoque (Quercus suber L.) desde los años 80 (Rupérez y Muñoz, 1980; Torres Juan, 1985). Igualmente se han identificado daños por decaimiento en masas mixtas de Quercus, habiéndose observado zonas afectadas por estos procesos dominadas por $Q$. pyrenica Willd., Q. faginea Lam., Q. canariensis Willd. y Q. coccifera L. (Carrasco et al., 2009).

Actualmente resulta difícil cuantificar la ingente cantidad de recursos económicos y humanos destinados a la búsqueda de posibles soluciones a este problema, $\sin$ que hasta la fecha se haya podido atajar el avance del decaimiento de las dehesas. Los principales avances se centran en el estudio de las causas de la podredumbre radical (Brasier et al., 1993; Sánchez et al., 2002; Navarro-Cerrillo et al., 2004, 2006; Ruiz Gómez et al., 2012, 2015) y en la aplicación de medidas preventivas y de limitación de daños al arbolado no afectado.

Respecto a la causa de la podredumbre radical, son numerosas las publicaciones que señalan al patógeno Phytophthora cinnamomi Rands. como principal factor desencadenante de los más graves episodios de decaimiento en dehesas extremeñas y andaluzas (Brasier et al., 1993; Brasier, 1996; Sánchez et al., 2002, 2003; NavarroCerrillo et al., 2004, 2006; Corcobado et al., 2013), así como en el montado portugués (Brasier et al., 1993, Sánchez et al., 2002, Moreira y Martins, 2005; Horta et al., 2010; Camilo-Alves et al., 2012).

Sin embargo, P. cinnamomi no es el único oomiceto capaz de producir podredumbre radical en dehesas y montados. Otras especies de pitiáceas han sido señaladas en ocasiones como agentes causales importantes en el decaimiento en encinares, como es el caso de Pythium spiculum Paul. (Romero et al., 2007), Phytophthora quercina Jung., Phytophthora psycrophila Jung. y Phytophthora syringae Kleb. (Pérez Sierra et al., 2013). En otras ocasiones, se han identificado más especies que actúan como patógenos oportunistas o acompañantes, que aparecen en cuadros sintomatológicos avanzados, agravando el decaimiento. Tal es el caso por ejemplo de Phytophthora gonapodydes (H.E. Petersen) Buisman. (Corcobado et al., 2010), Phytophthora dreschleri Tucker., Phytophthora cryptogea Pethybr. \& Laff. (Sánchez et al., 2005), Pythium irregulare Buisman., ó Phytophthora megasperma Drechsler. (Pérez Sierra et al., 2013) entre otras.

Hansen (2015) llama la atención sobre la influencia de la complejidad de los sistemas forestales en la diversidad de oomicetos de podredumbre radical en el suelo, señalando la importancia del efecto agregativo de la interacción de dichos organismos en el suelo, y la influencia del intercambio de materiales forestales de reproducción en estos procesos. Recurrentemente se describen nuevos efectos negativos de pitiáceas invasivas sobre especies vegetales, como resultado de campañas intensivas de reforestación (Oßwald et al., 2014).

La evaluación de la relación entre la diversidad de patógenos y la incidencia de los procesos de mortalidad de especies forestales debe focalizarse en el estudio de los 
síntomas y los grados de afección del arbolado, y en el análisis detallado de posibles relaciones con los agentes causales, considerando la presencia de diversas especies pitiáceas de podredumbre radical sobre la misma masa arbolada. Se asume que $P$. cinnamomi puede ser causa desencadenante de la podredumbre radical en la encina, por las evidencias aportadas de su patogenicidad sobre Q. ilex (Gallego et al., 1999), y los experimentos de inoculación realizados sobre plantones (Sánchez et al., 2005). Sin embargo, no se puede obviar el hecho de que, bajo las condiciones ambientales adecuadas, un complejo de especies de oomicetos causantes de podredumbre radical, sería capaz de reproducir los síntomas típicos causados por dicha especie.

El objetivo de este trabajo es evaluar la presencia de especies de pitiáceas causantes de podredumbre radical, diferentes de $P$. cinnamomi, en encinas de dehesas, en focos con síntomas evidentes de decaimiento.

\section{Material y métodos}

\subsection{Localizaciones}

Entre el otoño de 2014 y la primavera de 2015, se evaluaron 10 dehesas de encinar con procesos de mortalidad del arbolado, identificadas como posibles zonas de

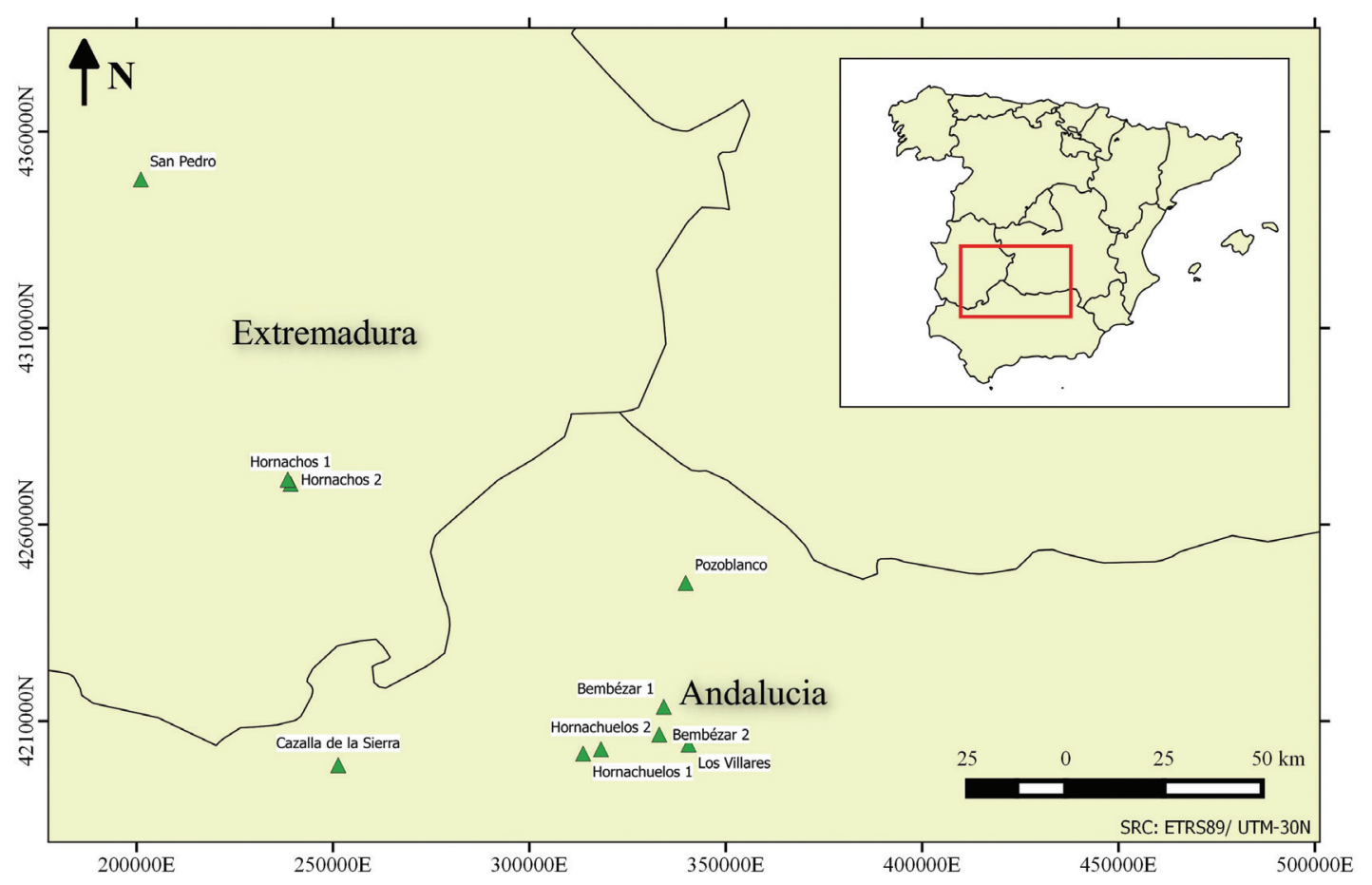

Figura 1. Mapa de situación de las localizaciones estudiadas. Líneas: Límites de comunidad autónoma. $\triangle$ : Centro de la localización. 
afección por podredumbre radical, en Andalucía y Extremadura (Figura 1). Los posibles focos presentes en cada dehesa se determinaron tras un diagnóstico visual de los daños, a través de la identificación de síntomas característicos de podredumbre radical (puntisecado, clorosis, defoliación de la copa, muerte súbita de pies aislados o la presencia de chancros y brotes epicórmicos en el tronco de pies afectados), así como la presencia de patrones espaciales típicos de localización y dispersión de árboles afectados.

\subsection{Evaluación de los focos y toma de muestras}

En cada uno de los focos de podredumbre radical se evaluó el daño en el arbolado mediante la adopción de una escala de defoliación asimilada de Ferreti (1994) en intervalos de 5\% respecto a un árbol con follaje completo en las condiciones locales (Comisión de las Comunidades Europeas, 1994), agrupándose los pies entre las clases 0 a 4 (Tabla 1), de acuerdo a los criterios de evaluación de la Red Europea de parcelas permanentes para el seguimiento intensivo y continuo de los ecosistemas forestales (Red de Seguimiento a gran Escala de Daños en los Bosques-Nivel I, 2013).

Tabla 1. Clases de defoliación de Ferreti (1994), en función del porcentaje de copa defoliada, y código asignado.

\begin{tabular}{lcc}
\hline Clase defoliación & \% defoliación & Código \\
\hline Defoliación Nula & $0-10 \%$ & 0 \\
Defoliación Ligera & $11-25 \%$ & 1 \\
Defoliación Moderada & $26-60 \%$ & 2 \\
Defoliación Grave & $>60 \%$ & 3 \\
Defoliación Total: & $100 \%$ & 4 \\
Árboles secos o muertos & & \\
\hline
\end{tabular}

Siguiendo las normas de la Red Europea, no se consideró defoliación las ramas secas por poda natural o la copa muerta, los huecos en la copa que nunca estuvieron cubiertos por ramas, y las hojas decoloradas en las que son frecuentes los tonos amarillos (Red de Seguimiento a gran Escala de Daños en los Bosques-Nivel I, 2013). Por el contrario, y según marcan dichas directrices, se consideró defoliación aquellos crecimientos que, debiendo portar hojas, carecieron de ellas (ramillos portadores), y las hojas secas en la copa con un color rojizo o marrón. De éste modo se consideró defoliación la copa cuando aparecían las hojas secas de manera homogénea, cubriendo los síntomas más característicos de la podredumbre radical, como son el puntisecado y la muerte súbita. 
Los focos fueron delimitados, identificando un núcleo donde los daños eran más acusados y, partiendo de la zona más afectada, hacia el exterior del mismo, estableciendo un perímetro de seguridad a partir de los últimos árboles afectados, de 2 filas de árboles con defoliación inferior al $25 \%$.

En cada foco se muestrearon al menos 2 árboles, el primero en la zona más afectada del foco, identificada como "nucleo", con grado alto de defoliación (en torno al 60\%). El segundo se seleccionó visualmente siguiendo el frente de avance del foco, en función de la dirección de la pendiente, la profundidad teórica de la capa freática y las posibles zonas de encharcamiento, y se muestreó un árbol con sintomatología de afección incipiente, generalmente con un grado de defoliación superior al $40 \%$ y con síntomas claros de daño con respecto al resto de árboles presentes a su alrededor, los cuales no presentaban síntomas de decaimiento. Se evitó seleccionar árboles evaluados como grado 4 de defoliación (secos, muertos o con defoliación $100 \%$, incluyendo los pies con síntomas de muerte súbita y copa totalmente atabacada). En total fueron muestreados 26 árboles (Tabla 2).

El análisis y la determinación de la presencia de patógenos de podredumbre radical se hizo a partir de una muestra de suelo, recogida bajo la copa de cada uno de los pies seleccionados según la metodología descrita por Zamora Rojas et al. (2014).

Tabla 2. Grado de defoliación estimado en los pies seleccionados para la obtención de muestras de suelo, con indicación de la localización a la que pertenece cada uno.

\begin{tabular}{|c|c|c|c|c|c|}
\hline Pie & $\begin{array}{c}\text { Grado } \\
\text { defoliación }\end{array}$ & Localización & Pie & $\begin{array}{c}\text { Grado } \\
\text { defoliación }\end{array}$ & Localización \\
\hline 26501 & 2 & Los Villares (Córdoba) & 141004_2 & 2 & Bembézar 1 (Córdoba) \\
\hline $26502 \mathrm{a}$ & 3 & Los Villares (Córdoba) & 141005_1 & 3 & Bembézar 2 (Córdoba) \\
\hline $26502 b$ & 1 & Los Villares (Córdoba) & 141005_2 & 2 & Bembézar 2 (Córdoba) \\
\hline 26503 & 0 & Los Villares (Córdoba) & 141005_B & 1 & Bembézar 2 (Córdoba) \\
\hline 140401 & 2 & Hornachos 1 (Badajoz) & 141007_1 & 3 & San Pedro (Cáceres) \\
\hline 140402_1 & 2 & Hornachos 2 (Badajoz) & 141007_2 & 2 & San Pedro (Cáceres) \\
\hline 140402_2 & 3 & Hornachos 2 (Badajoz) & 141007_3 & 1 & San Pedro (Cáceres) \\
\hline $140402 \_3$ & 2 & Hornachos 2 (Badajoz) & 141008_1 & 3 & Cazalla de la Sierra (Sevilla) \\
\hline 141001_1 & 3 & Hornachuelos 1 (Córdoba) & 141008_2 & 3 & Cazalla de la Sierra (Sevilla) \\
\hline 141001_2 & 2 & Hornachuelos 1 (Córdoba) & 141009 & 2 & Cazalla de la Sierra (Sevilla) \\
\hline 141003_1 & 3 & Hornachuelos 2 (Córdoba) & 141010_1_1 & 2 & Pozoblanco (Córdoba) \\
\hline $141003 \_2$ & 2 & Hornachuelos 2 (Córdoba) & 141010_1_2 & 1 & Pozoblanco (Córdoba) \\
\hline 141004_1 & 3 & Bembézar 1 (Córdoba) & 141010_4 & 1 & Pozoblanco (Córdoba) \\
\hline
\end{tabular}

\subsection{Aislamiento e identificación de patógenos}

El aislamiento e identificación de patógenos se llevó a cabo a partir del aislamiento de colonias en trampa vegetal mediante "soil baiting", y el cultivo por dupli- 
cado en medios selectivos a base de antibióticos NARPH y PARPBH, específicos para oomicetos de los géneros Pythium spp. y Phytophthora spp, según la metodología descrita por Ferguson y Jeffers (1999) y Jeffers y Martin (1986). En los casos en los que inicialmente se aislaron un gran número de especies del género Pythium spp., se realizó un segundo análisis tras un tratamiento de secado durante 5 días a temperatura ambiente y humidificación durante $48 \mathrm{~h}$, con el fin de eliminar propágulos de las especies más sensibles al estrés hídrico, y aumentar así la probabilidad de detección de especies poco competitivas en ambiente saprófito debido a una disminución de la competencia por las trampas vegetales, como es el caso de P. cinnamomi (Zamora Rojas et al., 2014).

La identificación de las especies del género Phytophthora se realizó tras la observación al microscopio de cultivos, y la identificación y caracterización de las estructuras vegetativas, de resistencia y reproductivas de los aislados en medios Carrot Agar (CA) y Potato Dextrose Agar (PDA), la proliferación y caracterización de estructuras reproductivas como esporangióforos y esporangios en "Soil Extract" (SE/PDA), así como, cuando fue necesario, mediante ensayos de "Mating Type" para observar las oosporas de especies heterotálicas, y el estudio de las curvas de crecimiento, siguiendo las indicaciones de Erwin y Ribeiro (1996).

La identificación de las especies del género Pythium spp. se llevó a cabo mediante la clasificación por caracteres de van der Plaat-Niterink (1981) una vez identificado el género mediante las claves de Erwin y Ribeiro (1996). Una vez encontrada la coincidencia taxonómica, se comprobó la determinación con el apoyo de las descripciones publicadas para cada una de las especies determinadas.

\subsection{Análisis estadístico}

Se comprobó la normalidad de los datos mediante el Test de Saphiro-Wilk, estableciendo el nivel de significación en el $5 \%(\mathrm{P}<0,05)$. La homocedasticidad se comprobó mediante el Test de homogeneidad de varianzas de Levene $(\mathrm{P}>0,05)$.

La presencia de diferencias significativas se estudió mediante un test de análisis de la varianza (ANOVA) $(\mathrm{P}<0,05)$. Para evaluar las diferencias entre las medias del número de aislamientos se utilizó el Test de Bonferroni $(\mathrm{P}<0,05)$, recomendado para agrupamientos con diferente número de observaciones (Turechek y McRoberts, 2013). El análisis se llevó a cabo mediante el software "R" (The R Foundation, Vienna, 2002).

\section{Resultados}

\subsection{Aislamiento de oomicetos}

En el conjunto de las 10 localizaciones muestreadas se identificaron 21 especies de oomicetos (Tabla 3), perteneciendo 5 de ellas a distintas especies del género Phytophthora spp. y 16 especies de Pythium spp. Además, se obtuvieron 5 aislados per- 
tenecientes a especies de Pythium spp. que no fue posible identificar mediante la metodología usada en el presente trabajo. El número máximo de especies identificadas en una misma muestra de suelo fue de 5 (Pythium heterothallicum Campbell \& Hendrix., Pythium flevoense van der Plaats-Niterink., Phytophthora citrophthora (R.E. Sm. \& E.H. Sm.) Sarej., Pythium adhaerens Sparrow y Pythium mamillatum Meurs.), en una muestra de la localización de Los Villares, en la cual también se determinó la presencia de $P$. cinnamomi.

Tabla 3. Especies de oomicetos identificadas en el conjunto de las parcelas. Las especies de Pythium spp. nombradas del 1 al 5, no fue posible identificarlas por caracteres morfológicos. $\mathrm{N}^{\circ}$ Identif.= Número de muestras de suelo en los que se identificó cada especie.

\begin{tabular}{|c|c|c|c|c|}
\hline Especie & $\begin{array}{c}\mathrm{N}^{0} \\
\text { Identif. }\end{array}$ & & & Referencia muestras positivas \\
\hline Phythophthora cactorum & 1 & 140402_1 & & \\
\hline Phytophthora cinnamomi & 8 & $260502 b$ & 141007_1 & 141007_2 141007_3 141004_1 141004_2 141003_1 141003_2 \\
\hline Phytophthora citropthora & 1 & 260501 & & \\
\hline Phytophthora cryptogea & 1 & 141003_1 & & \\
\hline Phytophthora gonapodydes & 1 & 141004_2 & & \\
\hline Pythium acanthophoron & 1 & 140009 & & \\
\hline Pythium adhaerens & 2 & 260501 & 141003_2 & \\
\hline Pythium apleroticum & 1 & 140401 & & \\
\hline Pythium aquatile & 2 & 141010_1_1 & 141003_1 & \\
\hline Pythium diclinum & 3 & 140401 & 140402_2 & 140402_3 \\
\hline Pythium flevoense & 1 & 260501 & & \\
\hline Pythium heterotalicum & 1 & 260501 & & \\
\hline Pythium irregulare & 6 & 141001_1 & 141001_2 & 141005_1 141005_2 141005_B 141004_2 \\
\hline Pythium mamillatum & 1 & 260501 & & \\
\hline Pythium oligandrum & 1 & 140009 & & \\
\hline Pythium papillatum & 1 & 141010_1_1 & & \\
\hline Pythium perniciosum & 2 & 141008_1 & 141008_2 & \\
\hline Pythium spiculum & 3 & $260502 b$ & 140401 & $140402 \_3$ \\
\hline Pythium spinosum & 1 & 140009 & & \\
\hline Pythium splendens & 1 & 140009 & & \\
\hline Pythium spp. 1 & 1 & $260502 \mathrm{a}$ & & \\
\hline Pythium spp. 2 & 1 & 260503 & & \\
\hline Pythium spp. 3 & 2 & 140402_1 & 140402_3 & \\
\hline Pythium spp. 4 & 2 & 141007_2 & 141007_3 & \\
\hline Pythium spp. 5 & 2 & 141007_2 & 141007_3 & \\
\hline Pythium tardicrescens & 1 & 141005_2 & & \\
\hline
\end{tabular}


En todos los focos de podredumbre radical, excepto uno (Pozoblanco, Córdoba), se aislaron especies de pitiáceas que han sido identificadas como agentes causales de podredumbre radical. En el caso de Pozoblanco, las especies identificadas fueron Pythium papillatum V.D. Matthews. y Pythium aquatile Höhnk. Del conjunto de muestras, sólo en 3 no se aisló ningún oomiceto, todas pertenecientes a la provincia de Córdoba, 2 en la localización de Pozoblanco y una en Los Villares.

\subsection{Diversidad de pitiáceas y podredumbre radical}

Phytophthora cinnamomi fue el patógeno que mayor número de aislamientos presentó, con 8 identificaciones positivas en muestras procedentes de 6 parcelas, pertenecientes a 4 localizaciones distintas (Tabla 2). La presencia de éste patógeno en las muestras no se relacionó significativamente con variaciones en la diversidad de pitiáceas aisladas $(\mathrm{F}=0,35 ; \mathrm{P}=0,71)$. Tampoco hubo relación entre la presencia del patógeno y el grado de daño de los pies $(\mathrm{F}=0,71 ; \mathrm{P}=0,41)$.

Los árboles con grado de defoliación "2" presentaron un mayor número de especies diferentes aisladas $(\mathrm{F}=3,43, \mathrm{p}<0,05)$ (Figura 2). En el conjunto, sólo un árbol con defoliación nula presentó un único aislamiento.

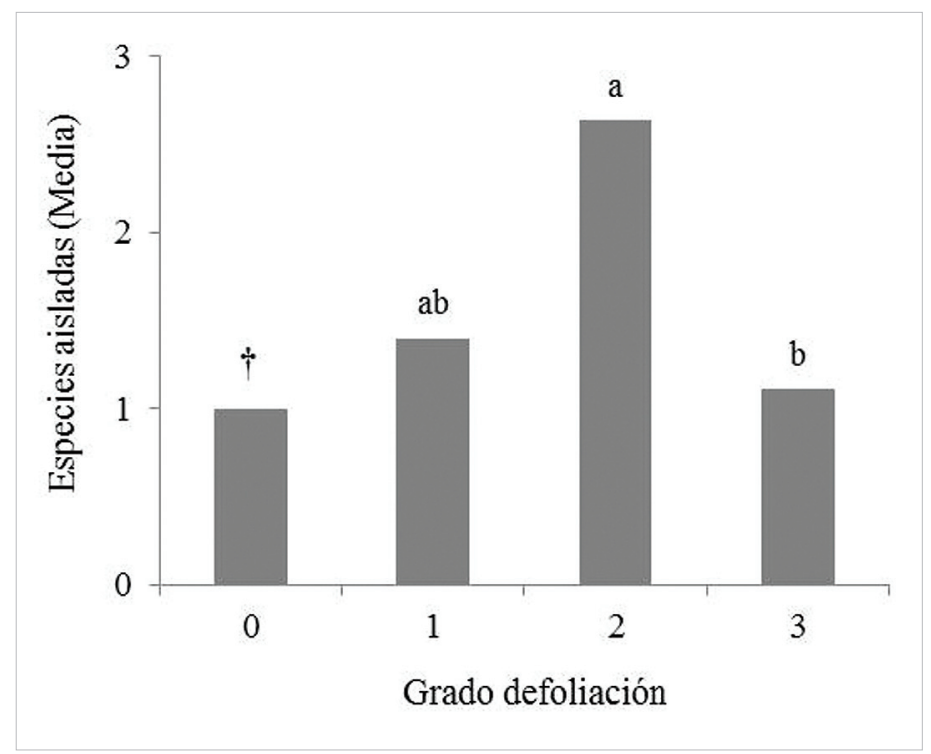

Figura 2. Número de especies identificadas por grado de daño del pie estudiado. Medias con la misma letra: No existen diferencias significativas entre las medias. $\uparrow$ : No incluido en el test de diferencias entre medias por ausencia de varianza $(n=1, X=1)$.

\section{Discusión}

\subsection{Especies aisladas}

La mayoría de las especies identificadas han sido descritas como agente causal de podredumbre radical en diferentes especies vegetales, algunas de carácter fores- 
tal y otras de carácter agronómico. Phytophthora cinnamomi es en la actualidad uno de los patógenos más estudiados de la familia Pythiaceae, junto con Phytophthora lateralis Tucker \& Milbrath. y Phytophthora ramorum Werres. (Hansen, 2015), y sin duda, causante de podredumbre radical en numerosas especies de interés forestal de los géneros Eucalyptus spp., Pinus spp. y Quercus spp. entre otros. P. citrophthora se ha identificado como agente causal de damping-off en plántulas de árboles y arbustos ornamentales (van der Plaat-Niterink, 1981). P. irregulare es un conocido agente de podredumbre radical de especies forestales (Eucalyptus spp. y Cupressus spp.), causando problemas relevantes en cultivos de invernadero (Katawczik, 2008). P. cryptogea es una especie muy extendida a nivel mundial, causante de podredumbre radical en numerosos cultivos leñosos (frutales) y en plantas ornamentales (van der Plaat-Niterink, 1981). Se ha comprobado la patogenicidad de $P$. gonapodyides, tanto en Q. suber (Jung et al., 1996; Balci y Halmschlager, 2003), como en Q. ilex (Corcobado et al., 2010).

Pythium flevoense y P. mamillatum son conocidos por causar enfermedades en especies cespitosas y gramíneas (van der Plaat-Niterink, 1981), mientras Pythium aquatile es un patógeno de raíz de actividad moderada, aunque ha sido aislado en muestras de Chamaecyparis spp., Rhododendron spp., Eucalyptus spp., y Prunus spp., con presencia de daños por podredumbre radical (Uzuhashi et al., 2008). Otros como Pythium tardicrescens Vanterpool., Pythium apleroticum Tokunaga., Pythium diclinum Tokunaga., o P. adhaerens no han sido descritos hasta la fecha como agentes causantes de podredumbre radical en especies leñosas, aunque sí en otras especies de gramíneas y leguminosas (van der Plaat-Niterink, 1981).

Otras especies aisladas no han sido descritas como agentes causantes de podredumbre radical. Pythium oligandrum Drechsler. y Pythium acanthophoron Sideris. son considerados micoparásitos, y no tiene actividad fitopatógena reconocida. Pythium oligandrum parasita principalmente ascomicetos, aunque se ha identificado como parásito de Pythium ultimum Trow. Algunos estudios indican que su presencia está relacionada con la inhibición del crecimiento de tricodermas (Lutchmeah y Cooke, 1984). Del mismo modo, se considera a la especie $P$. acanthophoron parásito del género Fusarium, aunque se ha reconocido su actividad como parásito de Pythium myriotylum Drechsler (Lodh y Webster, 1990).

\subsection{Causas del decaimiento}

En todas las localizaciones analizadas, excepto en el caso de Pozoblanco, se puede determinar que la causa principal de los daños sobre el arbolado está relacionada con la podredumbre radical causada por un complejo de oomicetos. En Pozoblanco se identificaron las especies Pythium aquatile y P. papillatum, aisladas bajo un pie que presentaba un grado moderado de afección (Grado "2"). Ambas especies son patógenos considerados moderadamente agresivos, y mayormente relacionados con gramíneas y especies agrícolas y ornamentales. Se podría atribuir su presencia al oportunismo, siendo la causa del decaimiento general del arbolado en éste caso diferente a la podredumbre radical. 
Los resultados obtenidos indican que los árboles donde se encontró un mayor número de especies causantes de podredumbre radical son aquellos que presentan daños en un grado incipiente o medio, reduciéndose la diversidad de especies patógenas presente en el suelo cuando el estado del árbol se agrava. Esta observación coincide con la apreciación de otros autores como Pérez Sierra et al. (2013), que apuntan que los árboles con un grado medio de afección son los más indicados para realizar el aislamiento de pitiáceas.

Un estado avanzado de daños en el arbolado, y un alto grado de defoliación, implican un bajo nivel de actividad de la planta, y por tanto una menor probabilidad de reposición de raíces finas. La inoculación de raíces con $P$. cinnamomi en especies de Quercus da lugar a una rápida detención del crecimiento apical (Cahill et al., 1989; Blaschke, 1994), y por otro lado se ha demostrado que éste patógeno coloniza la planta a través de las raíces finas (Ruiz Gómez et al., 2015). El análisis conjunto de ambas evidencias podría explicar la menor presencia de inóculo en aquellos pies muertos o con un estado de decaimiento terminal, donde la cantidad de raíces finas absorbentes $(<2 \mathrm{~mm}$ diámetro) y su proliferación se encuentran limitadas.

La presencia de $P$. cinnamomi en las muestras no se relacionó con una variación en el grado de afección en el arbolado, ni en la diversidad de oomicetos aislados. En éste trabajo, las parcelas se seleccionaron en función de la presencia de daños, y todos los árboles muestreados, excepto uno (Tabla 2), se encontraban afectados en mayor o menor medida, presentando un grado de defoliación mayor o igual a 1 . No se discute aquí sobre la incidencia de $P$. cinnamomi en el empeoramiento de las condiciones fitosanitarias de los pies donde se encuentra ésta especie, pero en éste trabajo no se puede concluir que los focos donde resultó un aislamiento positivo de dicha especie sufrieran un grado de decaimiento mayor que en el resto de localizaciones donde no se consiguió aislar dicha especie.

En siete de las diez fincas muestreadas, los focos se encontraban dentro de áreas reforestadas, o próximas a una repoblación, y en un caso, la masa de encinar se encontraba en una zona ajardinada donde se habían conservado los pies de encina de la masa anterior a la construcción del área recreativa. En el presente trabajo no se pudo discriminar la influencia de la localización de los focos en áreas repobladas, recreativas o cercanas a éstas, aunque muchas de las especies identificadas han sido descritas en trabajos con plantas ornamentales o en cultivos agronómicos. La presencia de dichas especies parece indicar que la afección del arbolado podría tener, en algunos casos, su origen en el comercio de plantas y el material de repoblación procedente de vivero, coincidiendo con las observaciones de otros autores (Oßwald et al., 2014; Hansen, 2015).

\section{Conclusiones}

La presencia de un mayor número de especies de oomicetos causantes de podredumbre radical en árboles con síntomas moderados de decaimiento, pone en evidencia que un complejo diverso de pitiáceas puede causar en el arbolado, bajo 
las condiciones apropiadas, los síntomas asociados a los procesos de mortalidad producidos por la podredumbre radical. Estos daños se dan en los focos muestreados con independencia del aislamiento de $P$. cinnamomi en las muestras analizadas.

La presencia de daños en el arbolado en aquellos focos donde se aislaron especies de pitiáceas diferentes a Phytophthora cinnamomi se pueden, por tanto, asociar a problemas de podredumbre radical. Es por ello, que el estudio de la mortalidad de encina (y otros Quercus) no debe focalizarse únicamente en la especie P. cinnamo$m i$. Las determinaciones que se practican rutinariamente en los laboratorios de patología vegetal deberían tener en cuenta la importancia de describir el mayor número de especies posible para realizar un mejor diagnóstico.

\section{Agradecimientos}

Los autores quieren agradecer el apoyo recibido por parte de la Consejería de Medio Ambiente y Ordenación del Territorio de la Junta de Andalucía, a través de la Agencia de Medio Ambiente y Agua (AMAYA), así como la ayuda y colaboración durante la realización del trabajo por IDAF y TSDehesa. El trabajo se ha realizado con la ayuda del Proyecto QUERCUSAT ("Detección temprana de procesos de decaimiento de Quercus ilex a partir de la integración de datos hiperespectrales y ecofisiológicos"), financiado por el Ministerio de Economía y Competitividad (CGL2013-40790-R). El primer autor es beneficiario de una Ayuda para la Formación del Profesorado Universitario (FPU13/00231) del Ministerio de Educación, Cultura y Deporte de España.

\section{Bibliografía}

Balci, Y., Halmschlager, E., 2003. Incidence of Phytophthora species in oak forests in Austria and their possible involvement in oak decline. Forest Pathology, 33:157-174. doi: 10.1046/j.1439-0329.2003.00318.x

Brasier, C.M., Robredo, F., Ferraz, J.F.P., 1993. Evidence for Phytophthora cinnamomi involvement in iberian oak decline. Plant Pathology, 42:140-145. doi: 10.1111/j.13653059.1993.tb01482.x

Camilo-Alves C.D.E.P., da Clara M.I.E., Ribeiro N.M.C.D., 2013 Decline of Mediterranean oak trees and its association with Phytophthora cinnamomi: a review. European Journal of Forest Research 132:411-432. doi: 10.1007/s10342-013-0688-Z

Carrasco, A., Fernández Cancio, A., Trapero Casas, A., López Pantoja, G., Sánchez Osorio, I., Ruiz Navarro, J.M., Jiménez Molina, J.J., Domínguez Nevado, L., Romero Martín, M.A., Carbonero Muñoz, M.D., Sánchez Hernández, M.E., Lucas Caetano, P.C., Gil Hernández, P., Fernández Rebollo, P., Navarro Cerrillo, R.M., Sánchez-Cuesta, R., Raposo Llobet, R., Rodríguez Reviriego, S., 2009. Procesos de Decaimiento Forestal (La Seca), Situación del Conocimiento. Ed. Consejería de Medio Ambiente, Junta de Andalucía, Córdoba. 
Comisión de las Comunidades Europeas, 1994. Reglamento 1091/94 de 29/04/94 (DOCE L125 de 18/05/94). En: Diario Oficial de las Comunidades Europeas, L 125 de 18/05/ 1994, pp 1-94.

Corcobado, T., Cubera, E., Pérez-Sierra, A., Jung, T., Solla, A., 2010. First report of Phytophthora gonapodydes involved in the decline of Quercus ilex in xeric conditions in Spain. New Disease Reports, 22:33. doi: 10.5197/j.2044-0588.2010.022.033

Corcobado, T.; Solla, A.; Madeira, M.A.; Moreno, G., 2013. Combined effects of soil properties and Phytophthora cinnamomi infections on Quercus ilex decline. Plant Soil 373: 403-13. doi: 10.1007/s11104-013-1804-z

Erwin, D.C. and Ribeiro, O.K., 1996. Phytophthora Diseases Worldwide. American Phytopathology Society, St Paul.

Ferguson, A.J. and Jeffers, S.N., 1999. Detecting multiple species of Phytophthora in container mixes from ornamental crop nurseries. Plant Disease 83:1129-1136. doi: 10.1094/ PDIS.1999.83.12.1129

Ferretti, M., 1994. Especies forestales mediterráneas. Guía para la evaluación de las copas. Publicaciones de la Comisión de las Comunidades Europeas. Comisión Económica de las Naciones Unidas para Europa. Bruselas, Ginebra.

Gallego, F.J., Pérez de Algaba, A., Fernández-Escobar, R., 1999. Etiology of oak decline in Spain. European Journal of Forest Pathology, 29:17-27. doi: 10.1046/j.1439-0329.1999. 00128.x

Hansen, E., 2015. Phytophthora species emerging as pathogens of forest trees. Current Forestry Reports, 1:16-24. doi: 10.1007/s40725-015-0007-7

Horta, M., Caetano, P., Medeira, C., Maia, I., Cravador, A., 2010. Involvement of the betacinnamomin elicitin in infection and colonisation of cork oak roots by Phytophthora cinnamomi. European Journal of Plant Pathology, 127:427-436. doi: 10.1007/s10658010-9609-x

Jeffers, S.N., Martin, S.B., 1986. Comparison of two media selective for Phytophthora and Pythium species. Plant Disease, 70:1038-1043. doi: 10.1094/PD-70-1038

Jung, T., Blasche, H., Neumann, P., 1996. Isolation, identification and pathogenicity of Phytophthora species from declining oak stands. European Journal of Forest Pathology, 26:253-272. doi: 10.1111/j.1439-0329.1996.tb00846.x

Katawczik, M., 2008. Pythium irregulare: PP728. Soilborne Plant Pathogens, NCSU Fall (http://www.cals.ncsu.edu)

Lodha, B.C., Webster, J., 1990. Pythium acanthophoron, a mycoparasite, rediscovered in India and Britain. Mycological Research, 94:1006-1008. doi: 10.1016/S0953-7562(09) 81323-3

Lutchmeah, R.S., Cooke, R.C., 1984. Aspects of antagonism by the mycoparasite Pythium oligandrum. Transactions of the British Mycological Society. 83:696-700. doi: 10.1016/S 0007-1536(84)80191-6

Moreira, A.C. and Martins, J.M.S., 2005. Influence of site factors on the impact of Phytophthora cinnamomi in Cork Oak Stands in Portugal. Forest Pathology, 35: 145-162. doi: 10.1111/j.1439-0329.2005.00397.x

Navarro-Cerrilllo, R.M., Fernández, P., Trapero, A., Caetano, P., Romero, M.A., Sánchez, M.E., Fernández, A., Sánchez, I., López, G., 2004. Los procesos de decaimiento de encinas y alcornoques. Dirección general de gestión del medio natural. Consejería de Medio Ambiente, Junta de Andalucía, Sevilla. 
Navarro-Cerrillo, R.M., Terán, A.I., Sánchez, M.E., 2006. Acción preventiva y curativa del fosfonato en el control de Phytophthora cinnamomi Rands. en encina y alcornoque. Boletin de Sanidad Vegetal y Plagas, 32:685-694.

Oßwald, W., Fleischmann, F., Rigling, D., Coelho, A.C., Cravador, A., Diez, J., Dalio, R.J., Horta Jung, M., Pfanz, H., Robin, C., Sipos, G., Solla, A., Cech, T., Chambery, A., Diamandis, S., Hansen, E., Jung, T., Orlikowski, L.B., Parke, J., Prospero, S., Werres, S., (2014). Strategies of attack and defence in woody plant-Phytophthora interactions. Forest Pathology, 44:169-190. doi: 10.1111/efp.12096

Pérez Sierra, A., López-García, C., León, M., García-Jiménez, J., Abad Campos, P., Jung, T., 2013. Previously unrecorded low-temperature Phytophthora species associated with Quercus decline in a Mediterranean forest in eastern Spain. Forest Pathology, 43: 331339. doi: 10.1111/efp.12037

Red de Seguimiento a gran Escala de Daños en los Bosques (Red de Nivel I), 2013. Manual de Campo. Área de Inventario y Estadísticas Forestales de la Dirección General de Desarrollo Rural y Política Forestal. Ministerio de Agricultura, Alimentación y Medio Ambiente. Madrid, 2013.

Romero, M.A., Sánchez, J.E., Jiménez, J.J., Belbarhi, L., Trapero, A., Lefort, L., Sánchez, M.E., 2007. New Pythium taxa causing root rot on mediterranean Quercus species in south-west Spain and Portugal. Journal of Phytopatology, 155:289-295. doi: 10.1111/j. 1439-0434.2007.01230.x

Ruiz Gómez, F.J., Sánchez de la Cuesta, R., Navarro Cerrillo, R.M., Pérez de Luque, A., 2012. A method to quantify infection and colonization of holm oak (Quercus ilex) roots by Phytophthora cinnamomi. Plant Methods, 8:39. doi: 10.1186/1746-4811-8-39

Ruiz Gómez, F.J., Navarro Cerrillo, R.M., Sánchez de la Cuesta, R., Pérez de Luque, A., 2015. Histopathology of infection and colonization of Quercus ilex fine roots by Phytophthora cinnamomi. Plant Pathology, 64:605-616. doi: 10.1111/ppa.12310

Rupérez, A., Muñoz, M., 1980. Grave enfermedad de las encinas. Boletin de Sanidad Vegetal y Plagas, 6:107-107.

Sánchez, M.E., Caetano, P., Ferraz, J., Trapero, A., 2002. Phytophthora disease of Quercus ilex in south-western Spain. Forest Pathology, 32:5-18. doi: 10.1046/j.1439-0329.2002. 00261.x

Sánchez, M.E., Sánchez, J.E., Navarro-Cerrillo, R., Fernández, P., 2003. Incidencia de la podredumbre radical causada por Phytophthora cinnamomi en masas de Quercus en Andalucía. Boletín de Sanidad Vegetal y Plagas, 29:87-108.

Sánchez, M.E., Andicoberry, S., Trapero, A., 2005. Pathogenicity of three Phytophthora spp. causing late seedling rot of Quercus ilex ssp. ballota. Forest Pathology, 35:115-125. doi: 10.1111/j.1439-0329.2004.00392.x

Torres Juan, J., 1985. El Hypoxilon mediterraneum (De Not.) Mill. Y su comportamiento en los encinares y alcornocales andaluces. Boletin del Servicio de Plagas, 11:185-191.

Turechek, W.W., McRoberts, N. 2013. Considerations of scale in the analysis of spatial pattern of plant disease epidemics. Annual Review of Phytopathology, 51:453-472. doi: 10.1146/annurev-phyto-081211-173017

Uzuhashi, S., Tojo, M., Kobayash, S., Tokura, K., Kakishima, M., 2008. First records of Pythium aquatile and P. macrosporum isolated from soils in Japan. Mycoscience 49:276-279. doi: 10.1007/S10267-008-0416-6

Van der Plaat-Niterink, A.J., 1981. Monograph of genus Pythium. Studies in Micology, 21. 
Zamora Rojas, E., Andicoberry, S., Sánchez, M.E., 2014. El decaimiento y la podredumbre radical en las dehesas andaluzas. En: Ecosistemas de dehesa: Desarrollo de políticas y herramientas para la gestión y conservación de la biodiversidad. Anexo A.1.IV. LIFE11/BIO/ES/000726 (www.uco.es/biodehesa). 